\title{
Participation of pulmonary embolism response teams during the perioperative period
}

\section{Participación de los equipos de respuesta rápida de tromboembolia pulmonar durante el perioperatorio}

\author{
Mateo Porres-Aguilar*, Javier E. Anaya-Ayala², Flavio A. Grimaldo-Gómez ${ }^{3}$, Luis E. Santos-Martínez, \\ David Jiménez ${ }^{5}$, Mateo Porres-Muñoz ${ }^{6}$, Raúl Izaguirre-Ávila ${ }^{3}$, and Raúl Carrillo-Esper ${ }^{7}$
}

${ }^{1}$ Division of Adult Thrombosis Medicine, Jewish General Hospital, Department of Medicine, McGill University, Montreal, Quebec, Canada, and Sociedad Mexicana de Trombosis y Hemostasia (SOMETH); ${ }^{2}$ Department of Angiology and Vascular Surgery, Instituto Nacional de Ciencias Médicas y Nutrición Salvador Zubirán; ${ }^{3}$ Department of Hematology, Instituto Nacional de Cardiología Ignacio Chávez, Sociedad Mexicana de Trombosis y Hemostasia (SOMETH); ${ }^{4}$ Department of Pulmonary Hypertension and Right Ventricle, Cardiology Hospital, CMN Siglo XXI, Instituto Mexicano del Seguro Social (IMSS), Mexico City, Mexico; ${ }^{5}$ Department of Respiratory Medicine, Hospital Ramón y Cajal and Universidad de Alcalá, Madrid, Spain; ${ }^{6}$ Beneficencia Española de Tampico, Department of Internal Medicine and Geriatrics, Tampico; ${ }^{7}$ Critical Areas Division, Instituto Nacional de Rehabilitación Guillermo Ibarra, Sociedad Mexicana de Trombosis y Hemostasia (SOMETH), Mexico City, Mexico

\begin{abstract}
Acute pulmonary embolism represents a frequent cause of cardiovascular morbidity and mortality, only exceeded by acute coronary syndromes and cerebrovascular disease. The start-up and implementation of a designated pulmonary embolism response team (PERT) are necessary to improve prognosis and minimize long-term sequelae in the subgroup of patients with significant pulmonary embolism. Herein, we describe and discuss an overview of the current and potential role of PERT, with a focus on the perioperative period.
\end{abstract}

Key words: Pulmonary embolism. Rapid response teams. Outcomes assessment. Mexico.

\section{Resumen}

La tromboembolia pulmonar aguda representa una causa frecuente de morbimortalidad cardiovascular, sólo rebasada por los síndromes coronarios agudos y la enfermedad cerebrovascular. El inicio y la intervención de un equipo multidisciplinario de respuesta rápida en la tromboembolia pulmonar son imperantes para mejorar el pronóstico y reducir al mínimo las posibles secuelas en el subgrupo de pacientes más graves. En este artículo de revisión se describe y revisa de manera general el papel actual y potencial que tienen dichos equipos de respuesta rápida, con un enfoque particular en el perioperatorio.

Palabras clave: Tromboembolia pulmonar. Equipos de respuesta rápida. Evaluación de resultados. México.

\section{Correspondence:}

*Mateo Porres-Aguilar

E-mail: mateo.porres@mail.mcgill.ca
Available online: $04-12-2020$

Date of reception: 16-01-2020

Date of acceptance: 26-03-2020

DOI: 10.24875/ACME.M20000132
Arch Cardiol Mex (Eng). 2020;90(3):294-300

www.archivoscardiologia.com license (http://creativecommons.org/licenses/by-nc-nd/4.0/). 


\section{Introduction}

Acute pulmonary thromboembolism (PTE) represents the third most common cause of cardiovascular mortality in the western world, after acute coronary syndromes and cerebrovascular disease ${ }^{1-5}$. Rapid and accurate appropriate classification and stratification of risk in acute PTE represent, without a doubt, the cornerstone for making clinical decisions in the therapeutic approach and prognosis. Mortality from intermediate-high (sub-massive) and high-risk PTE remains high. High risk, acute PTE, formerly known as massive PTE, is clinically defined as frank hemodynamic instability, manifested by persistent hypotension (systolic blood pressure $<90 \mathrm{mmHg}$ or cardiogenic shock) and has a mortality of $30-50 \%$.

\section{Importance of acute PTE current classification and risk stratification}

Recently, the European Society of Cardiology (ESC) clinical guidelines proposed an important adjustment within the classification of intermediate-risk acute PTE by subdividing patients at intermediate-high and intermediate-low risk ${ }^{4}$. The first subgroup is characterized by objective right ventricular dysfunction and myocardial damage markers elevation (e.g., troponin and brain natriuretic peptide elevation); the second is characterized by having just one of the above-mentioned parameters or none of them. Intermediate-high-risk acute PTE it has a mortality of $10-25 \%$. 7,8 . At present, there is an important controversy in multiple medical societies and among clinical researchers themselves on how to improve intermediate-high-risk acute PTE definition, given the different heterogeneous definitions in various studies and clinical protocols over the last decade. Based on the above, it is imperative and necessary to formulate soon a uniform and homogeneous definition based on a consensus supported by a global group of experts in acute PTE, just as a universal definition of acute myocardial infarction exists and has been validated and is constantly evolving, with the common purpose of being able to identify the subgroup of patients with intermediate-high-risk acute PTE that actually is severe or patients with poorer short-term prognosis (e.g., mortality or 30-day venous thromboembolism recurrence) or in-hospital torpid clinical evolution with progressive clinical deterioration despite having initiated hospitalization in a "normotensive" state with early anticoagulation implementation $(<12 \mathrm{~h}$ after admission to the emergency room).
The proposal to carry out a proper, fast, and accurate multimodal approach to risk stratification and prognosis is highly important, and it should include clinical criteria, clinicians' intuition regarding the suspicion of rapid and progressive clinical deterioration, aided and supported by clinical scales of acute PTE severity, objective demonstration of right ventricular dysfunction by echocardiography or chest contrast-enhanced computed tomography, and elevated biomarkers of myocardial damage ${ }^{9}$. PTE rapid response teams (PTERRTs) have the capability to complete, analyze, and integrate said combined risk stratification, and their purpose is being able to early detect the most critically ill patients and those who actually have a high short-term mortality within the hospitalization period, which entails a worse prognosis; this way, a personalized/ individualized therapeutic approach plan can be devised according to each patient's particular characteristics, as well as for the detection of patients in whom a more radical therapeutic modality is necessary, for example, the need for early reperfusion procedures ${ }^{10}$.

\section{Fundamental reasons for PTERRTs creation, organization, and operation}

The Massachusetts General Hospital (MGH), in Boston, Massachusetts, introduced PTERRTs in 2012 ${ }^{11-15}$. Subsequently, other US tertiary care centers, both academic, and community-based began to form, organize, structure, and incorporate their respective PTERRTs. In May 2015, the National Pulmonary Embolism Response Teams (PERTs) Consortium was founded in the US, with the primary objectives of continually promoting the PERT model, guiding each team leaders to practice clinical care with excellence, as well as the diagnostic and therapeutic approach and prognosis, to generate a robust database for clinical research and multicenter studies that would strengthen evidence-based medicine for acute PTE care updating, as well as to create and apply quality improvement programs for patients with severe and complex acute therapeutic plasma exchange (TPE) ${ }^{16,17}$. At present, there are already more than 140 academic and hospital institutions in the USA and the entire world affiliated to and accredited by the PERT Consortium ${ }^{16,17}$.

\section{PTERRTs structure and operation}

Usually, PTERRTs are made up of a multidisciplinary team of various specialties, with a particular common interest in venous thromboembolism and mainly include vascular medicine and adult thrombosis, emergency 
medicine, internal medicine, invasive cardiology and diagnostic and interventional vascular radiology, pulmonology/intensive care, as well as cardiothoracic and vascular surgery specialists. It should be emphasized that not in all cases should all of the above-mentioned specialties be available; actually, depending on the resources of each hospital institution, the number of members of a PTERRT can range from two to three experts up to a larger team ranging from eight to ten. Subsequently, the main members of a PTERRT are mnemonically summarized using the acronym for PERT:

- $\mathrm{P}=$ Pulmonologist/intensivist (pulmonology and intensive therapy)

- E = Endovascular expert (cardiology or interventional radiology)

$-\mathrm{R}=$ Diagnostic radiology

$-T$ = Thrombosis expert (vascular medicine, adult thrombosis or hematology).

One of the PTERRT main purposes is to quickly, effectively, accurately, efficaciously, and in an agreement between the team members, facilitate the formulation/ issuance of a therapeutic plan and optimal, reasonable, and personalized care provision for a specific patient with severe complex acute PTE (intermediate-high and high risks), after evaluation and risk analysis with regard to the benefit within the existing alternatives and therapeutic modalities, especially when the possible use of measures of early reperfusion is considered in patients with cardiogenic shock or with the possibility of rapid progressive deterioration within the ensuing 24-48 $\mathrm{h}^{18}$.

Any doctor or trainee resident can activate the code and consultation for PTERRTs within or outside the hospital; the resident or specialist doctor who are on duty exclusively for consultations related to PTERRTs should evaluate, analyze, and integrate said consultation in a period no longer than $90 \mathrm{~min}$; subsequently, the case is presented and reviewed among the team members, a conclusion is drawn, and clinical decisions are made regarding the treatment and prognosis by consensus between the team members, and the consultant physician is immediate, in real-time and directly notified.

Table 1 lists the main advantages (pros) and possible obstacles (cons) within PTERRTs operation and performance.

\section{PTERRTs initial results}

Given the significant advances in science, the knowledge and technology about therapeutic measures for reperfusion in acute TPE, such as systemic thrombolysis plus fibrin-specific agents, regular catheter-directed or ultrasound-assisted thrombolysis devices, aspiration or catheter-assisted percutaneous mechanical thrombectomy, open surgical pulmonary embolectomy and advanced hemodynamic support, and multidisciplinary communication between several medical specialties and surgical procedures based on teamwork are required, which has markedly grown ${ }^{11-15}$.

Based on the above, and along with the advent, fast growth, development, and constant evolution of PTERRTs, some of the early studies that best characterize and describe PTERRTs performance are described below.

Kabrhel et al. ${ }^{13}$ retrospectively described Boston's MGH PTERRTs performance in 394 patients in whom PTERRTs were activated, in 217 patients $(69 \%)$, anticoagulation was recommended, in $28(9 \%)$, catheter-assisted thrombolysis, in $15(8 \%)$, systemic thrombolysis, and in $8(3 \%)$, surgical pulmonary embolectomy. Fifty-three percent of PTERRT consultations or activations took place at night, and on weekends, PTERRTs acceptance and adoption continuously increased, with an increase in the number of activations of $16 \%$ every 6 months. All-cause mortality was $12 \%$ in patients with acute PTE objective demonstration.

The New York University group published in 2017 its experience with 124 PRERRT activations in 20 months ${ }^{19}, 90.8 \%$ of their patients belonged to the intermediate-high-risk acute PTE subgroup, and $9.2 \%$ to high-risk acute PTE. Among the reperfusion treatments, catheter-assisted thrombolysis was used in 25 patients $(20 \%)$, systemic thrombolysis in $6(5 \%)$, and anticoagulation only in 54 (44\%). In-hospital mortality of $13 \%$ was recorded, with five major bleeding episodes: one patient who received catheter-assisted care, one patient who underwent systemic thrombolysis, and three only under anticoagulation.

Recently, Rosovsky et al. ${ }^{20}$ published a 10-year retrospective analysis, which evaluated the therapeutic and prognostic patterns in patients with severe acute PTE (high-intermediate and high risk), comparing the pre-PTERRT (2006-2012) with the post-PTERRT era (2012-2016); more patients underwent an early radical reperfusion therapeutic behavior (1\% vs. $14 \%$; $p \leq 0.0001)$ during the post-PTERRT era; however, there was no significant difference in the number of major or fatal bleeding episodes as well as with regard to the difference in all-cause mortality.

Also recently, the first multicenter retrospective PTERRT analysis was published in USA, which included 418 documented patients of the US PERT Consortium active registry from October 2016 to October 2017; the number of monthly activations that included 
Table 1. Advantages and obstacles during the creation, organization, and operation of pulmonary thromboembolism rapid response teams (PTERRTs)

Advantages (PROS)

- PTERRTs generate a highly valuable plan with regard to the most appropriate diagnostic and therapeutic recommendations for complex cases of severe acute PTE

- They bring together a multidisciplinary team of experts, which critically reviews and analyzes the risk/benefit with regard to the various therapeutic and care alternatives until reaching a consensus in the vast majority of consulted cases

- They effectively facilitate the mobilization of diagnostic and therapeutic resources within a hospital institution

- They proactively participate in the in-hospital treatment of patients with severe acute PTE, including appropriate selection of long-term anticoagulation regimens, and coordinate appropriate outpatient follow-up

- They sustain and support the strengths of each subspecialty that intervenes in said teams to promote PTERRTs cohesion

- They generate a large clinical and epidemiological database in favor of clinical research and continuous improvement of in-hospital quality in the care of patients with venous thromboembolism

- They identify groups of particular interest in venous thromboembolism within existing subspecialties in educational/academic hospital institutions

- They accept and globally/worldwide adopt PTERRTs in hospitals and academic and private institutions

- They favor the possible change in the paradigm of diagnostic and therapeutic approach in acute PTE, especially in its most severe forms

Obstacles/challenges (CONS)

- Lack of interest and commitment, which results in major failure during the construction and initiation or operation of a PTERRT

- A consensus or final decision is not reached among the specialists that make up a PTERRT; the consequence is a significant delay in the most appropriate therapeutic approach, especially for all patients who are in critical condition

- Barriers or obstacles during the diffusion and expansion of the PTERRT concept, whether internally (within the hospital or academic institution where said team is initiated) or externally (in other local or regional hospitals)

- False perception that PTERRTs were created only for excessive use or abuse of endovascular procedures in the treatment of severe acute PTE

- Lack of collaboration, interest, or commitment by trainee residents (e.g., emergency department personnel, internists, pulmonologists, cardiologists, or hematologists) as potential leaders and proactive participation in a PTERRT

- Aspects to be solved, such as adequate financial compensation and remuneration when formulating an appropriate diagnostic and therapeutic approach plan by a PTERRT

PTERRTs ranged from 3 to 13 monthly consultations; most activations occurred in patients with intermediate-low and intermediate-high-risk PTE, with $34 \%$ and $35 \%$, respectively, and $12 \%$ of these activations occurred in patients with high-risk/massive PTE. There was considerable variability in the use of reperfusion measures between different PTERRTs (16 and 44\%), with a 30 -day mortality of $16 \% 21$.

Figure 1 is a flowchart that comprehensively outlines PTERRTs operation.

\section{Potential role of PTERRTs in the perioperative period}

Given the recent introduction of PTERRTs in clinical daily practice, there is still no solid evidence supported by medical publications analyzing the potential role of PTERRTs in the treatment of complicated patients in the perioperative period.

It is important for PTERRTs to have a collaborative and coordinated work with specific departments appointed in thrombosis medicine; the latter has been developed in some countries such as Canada. PTERRTs collaboration in perioperative pharmacological treatment with direct oral anticoagulants such as vitamin $\mathrm{K}$ antagonists could possibly be considered as a future perspective since perioperative management of these anticoagulants can be quite complicated and confusing for most doctors. The following is important questions: is it necessary to discontinue oral anticoagulation for any procedure or operation? If the answer is affirmative, when is it convenient doing it? Is perioperative 


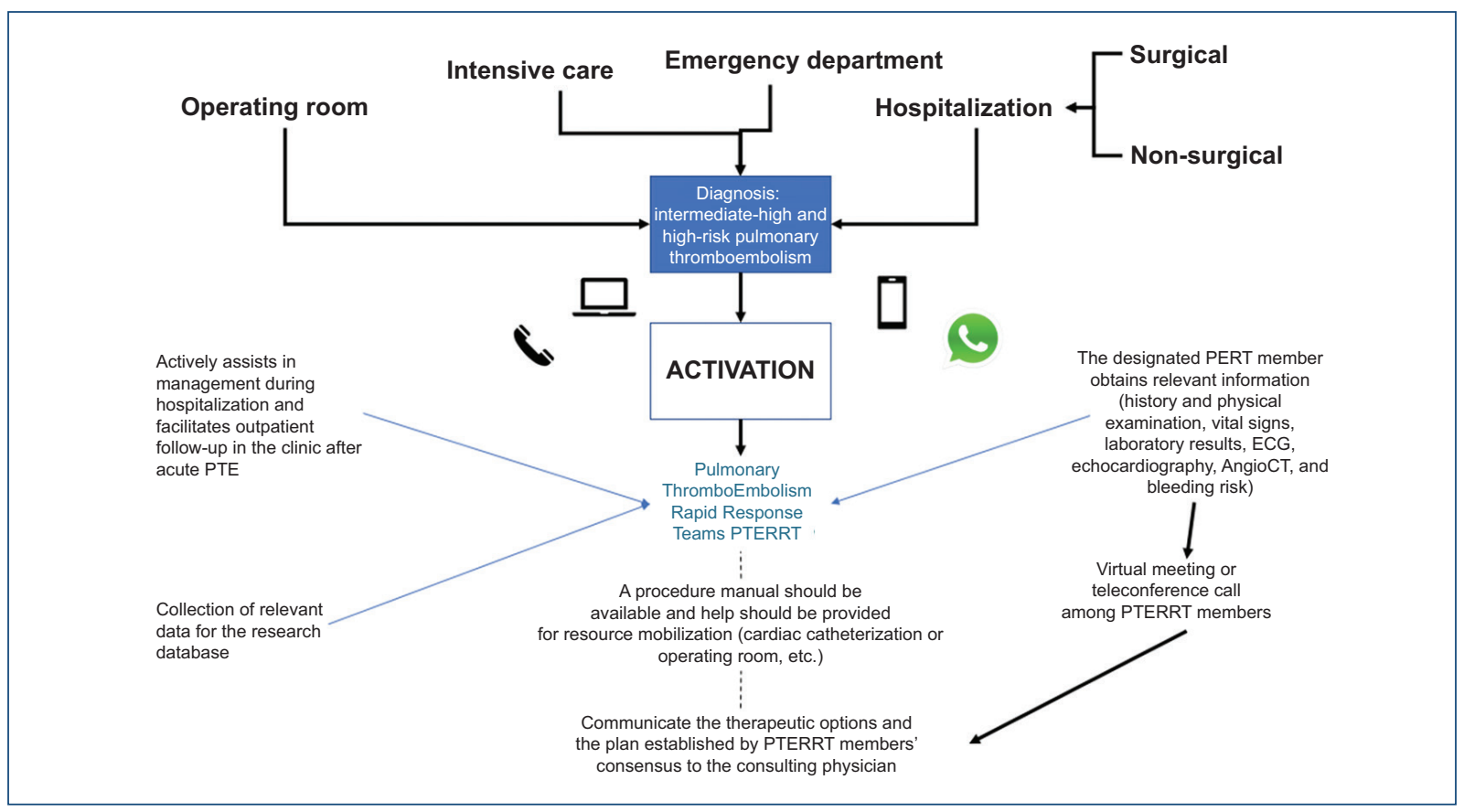

Figure 1. Flowchart comprehensively outlining PTE rapid response teams functioning.

adjustment required, such as anticoagulation regimen bridging in patients taking Vitamin $\mathrm{K}$ antagonists? When is it convenient to resume anticoagulation in the post-operative period? ${ }^{22,23}$ These questions could be addressed in PTERRTs, in close interrelation also with pharmaceutical chemists with experience in anti-thrombotic agents, who should also be viewed as PTERRTs proactive members.

PERRTs could take part in the perioperative assessment of thromboembolic and hemorrhagic risk in medical and surgical patients, either in previously anticoagulated subjects or in complicated individuals in whom an assessment focused on appropriate in-hospital thromboprophylaxis is required, as well as when there is the need for pharmacological thromboprophylaxis prolongation beyond hospital discharge (e.g., 30-45-day extended pharmacological thromboprophylaxis), should it be indicated based on thromboembolic risk scales.

Another of the indisputable and valuable activities, in which PTERRTs can be a cornerstone, is that related to rapid, correct, and efficient risk stratification, in addition to the detection of significant risk factors that modify morbidity and mortality in patients diagnosed with perioperative severe acute PTE. The Japanese Society of Anesthesiology study group recently described, in a retrospective analysis of 214 patients, significant perioperative risk factors associated with an elevated 30-day mortality, including the following: male gender, advanced older age $>80$ years, prolonged immobilization, history of heart failure, and no previous history of anticoagulant consumption ${ }^{24}$. Table 2 proposes PTERRTs potential functions in the perioperative period.

The treatment of patients with severe complicated acute PTE is a common cause of controversy due to the heterogeneity of patients, availability of therapeutic modalities and resources each hospital institution has, absence of consensuated guidelines and sometimes due to a lack of specialists at secondary care. Although the introduction of PTERRTs has emerged as an standardized approach, the effect thereof is so far not entirely clear. In a recent study, Chaudhury et al. conducted a retrospective analysis that compared the results 18 months before and after the introduction of PTERRTs; the authors observed, in a cohort of 769 patients, a significant decrease in 30-day in-hospital mortality, which was more pronounced in subjects with intermediate-high and high risk (before PTERRTs, $10.0 \%$ vs. after PTERRTs introduction, $5.3 \% ; p=0.02$ ), which was highly noticeable in patients who underwent some type of early reperfusion treatment (e.g., systemic thrombolysis or 
Table 2. Potential functions of pulmonary thromboembolism rapid response teams (PTERRTs) in the perioperative period

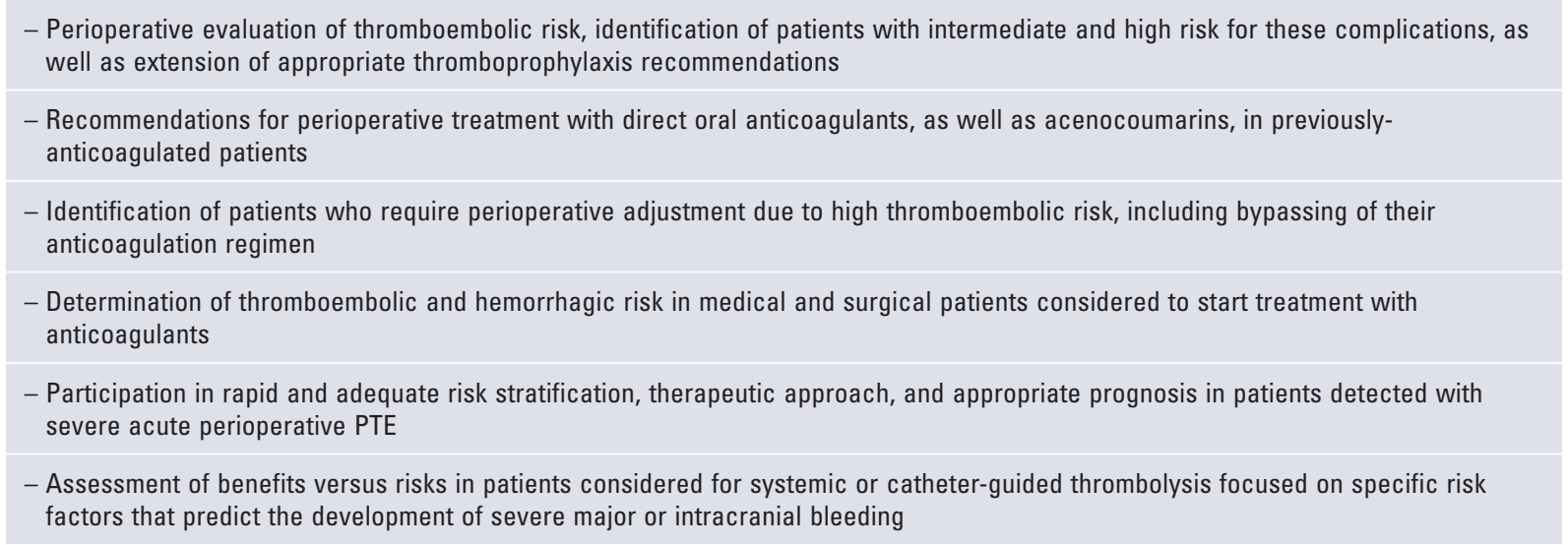

catheter-directed endovascular therapy), suggesting that this population is the most benefited from PTERRTs ${ }^{25}$.

There are still many contrasting results between various PTERRT groups; however, adequate utilization, accumulation of experience through the presentation of cases, as well as consistency in the excellence of patient care with venous thromboembolism by PTERRTs, without a doubt, will show its positive effects on future medium and long term morbidity and mortality in acute PTE, particularly in intermediate-high and high-risk groups.

\section{Conclusions}

PTERRTs were designed to offer, based on a detailed and multidisciplinary evaluation, an optimal diagnostic, therapeutic, and prognostic plan in complicated patients with severe acute PTE, in a personalized form for each clinical scenario.

These teams represent not only a concept, but a unique, authentic, and innovative process in constant development and evolution, the main purpose of which is to be adopted, not only in the USA but throughout the world ${ }^{26}$. As more robust scientific evidence accumulates, and there is better performance of said teams, a significant positive effect will be possible to reduce the elevated mortality seen in patients with intermediate-high and high-risk acute PTE; this way, quality of life and prognosis will be improved, and the paradigm in the care of this devastating disease will be changed.

\section{Funding}

None.

\section{Conflicts of interests}

The authors declare that they have no conflicts of interest.

\section{Ethical disclosures}

Protection of human and animal subjects. The authors declare that no experiments were conducted on humans or animals for this investigation.

Confidentiality of data. The authors declare that no patient data appear in this article.

Right to privacy and informed consent. The authors declare that no patient data appear in this article.

\section{References}

1. Teleb M, Porres-Aguilar M, Anaya-Ayala JE, Rodríguez-Castro C, Porres-Muñoz M, Mukherjee D. Potential role of systemic thrombolysis in acute submassive intermediate risk pulmonary embolism: review and future perspectives. Ther Adv Cardiovasc Dis. 2016;10(2):103-110.

2. Go AS, Mozaffarian D, Roger VL, Benjamin EJ, Berry JD, Blaha MJ, et al. Executive summary: heart disease and stroke statistics-2014 update: a report from the American Heart Association. Circulation. 2014; 129(3):399-410.

3. Cohen AT, Agnelli G, Anderson FA, Arcelus JI, Bergqvist D, Brecht JG, et al. Venous thromboembolism (VTE) in Europe. The number of VTE events and associated morbidity and mortality. Thromb Haemost. 2007:98(4):756-64.

4. Konstantinides SV, Torbicki A, Agnelli GC, Danchin N, Fitzmaurice D, Galie N, et al. 2014 European Society of Cardiology (ESC) guidelines on the diagnosis and management of acute pulmonary embolism. Eur Heart J. 2014;35(43):3033-3069.

5. Mahan CE, Borrego ME, Woersching AL, Federici R, Downey R. Venous thromboembolism: annualised United States models for total, hospital-acquired and preventable costs utilising long-term attack rates. Thromb Haemost. 2012;108(2):291-302.

6. Porres-Aguilar M, Anaya-Ayala JE, Heresi GA, Rivera-Lebron BN. Pulmonary embolism response teams: a novel approach for the care of complex patients with pulmonary embolism. Clin Appl Thromb Hemost. 2018;19:1076029618812954. doi: 10.1177/1076029618812954.

7. Jiménez D, Bikdeli B, Marshall PS, Tapson VF. Aggressive treatment of intermediate-risk patients with acute symptomatic pulmonary embolism. Clin Chest Med. 2018;39(3):569-581. 
Arch Cardiol Mex (Eng). 2020;90(3)

8. Kearon $\mathrm{C}$, Akl EA, Ornelas J, Blaivas A, Jiménez D, Bounameaux $\mathrm{H}$, et al Antithrombotic therapy for VTE disease: CHEST Guideline and Expert Panel Report. Chest. 2016;149(2):315-352.

9. Kosova EC Desai KR Schimmel DR. Endovascular management of massive and submassive acute pulmonary embolism: current trends in risk stratification and catheter-directed therapies. Curr Cardiol Rep. 2017; 19(6):54.

10. Porres-Aguilar M, Anaya-Ayala JE, Santos-Martínez LE. Intermediate-high risk pulmonary embolism: redefining it better with the support of the pulmonary embolism response teams approach. Arch Cardiol Mex. 2019;89(1):53-55.

11. Provias T, Dudsinzki DM, Jaff MR, Rosenfield K, Channick RN, Baker J, et al. The Massachusetts General Hospital Pulmonary Embolism Response Team (MGH PERT): creation of a multidisciplinary program to improve care of patients with massive and submassive pulmonary embolism. Hosp Pract. 1995;2014;42(1):31-37.

12. Dudzinski DM, Giri J, Rosenfield K. Interventional therapies for pulmonary embolism. Circ Cardiovasc Interv. 2017;10(2).pii: e004345. doi: 10.1161/ CIRCINTERVENTIONS.116.004345.

13. Kabrhel C, Rosovsky R, Channik RN, Jaff MR, Weinberg I, Sundt T, et al. A multidisciplinary pulmonary embolism response team: initial 30-month experience with a novel approach to delivery of care to patients with submassive and massive pulmonary embolism. Chest. 2016;150(2):384-393.

14. Witkin AS. Acute and chronic pulmonary embolism: the role of the pulmonary embolism response team. Curr Opin Cardiol. 2017;32(6):672-678.

15. Witkin AS, Harshbarger S, Kabrhel C. Pulmonary embolism response teams. Semin Thromb Hemost. 2016;42(8):857-864. Epub 2016 Oct 21. Review.

16. Available from: https://pertconsortium.org/about/(Accessed on July 15, 2019)

17. Serhal M, Haddadin IS, Heresi GA, Hornacek DA, Shishebhor MH, Bartolomew JR. Pulmoanry embolism response teams. J Thromb Thrombolysis. 2017;44(1):19-29.
18. Teleb M, Porres-Aguilar M, Rivera-Lebron BN, Ngamdu KS, Botrus G Anaya-Ayala JE, et al. Ultrasound-assisted catheter-directed thrombolysis: a novel and promising endovascular therapeutic modality for intermediate-risk pulmonary embolism. Angiology. 2017;68(6):494-501.

19. Sista AK, Friedman OA, Dou E, Denvir B, Askin B, Stern J, et al. A pulmonary embolism response team's initial 20-month experience treating 87 patients with submassive and massive pulmonary embolism. Vasc Med. 2018;23(1):65-71.

20. Rosovsky R, Zhao K, Sista A, Rivera-Lebron B, Kabrhel C. Pulmonary embolism response teams: purpose, evidence for efficacy, and future research directions. Res Pract Thromb Haemost. 2019;3(3):315-330.

21. Schultz J, Giordano N, Zheng H, Parry BA, Barnes GD, Heresi GA, et al. EXPRESS: a multidisciplinary pulmonary embolism response team (PERT) - Experience from a national multicenter consortium. Pulm Circ. 2019:2045894018824563. doi: 10.1177/2045894018824563. [Epub ahead of print

22. Leitch J, van Vlimen J. Managing the perioperative patient on direct ora anticoagulation. Can J Anesth. 2017:64:656-672.

23. Barnes GD, Mouland E. Peri-procedural management of oral anticoagulants in the DOACs era. Prog Cardiovasc Dis. 2018;60:600-606.

24. Working group for audit about perioperative accident and pulmonary embolism of Japanese Society of Anesthesiologists. Perioperative risk factors for death among patients with symptomatic pulmonary thromboembolism. J Anesth. 2017;31(3):478-482.

25. Chaudhury P, Gadre SK, Schneider E, Renapurkar RD, Gomes M, Haddadin I, et al. Impact of multidisciplinary pulmonary embolism response team availability on management and outcomes. Am J Cardiol. 2019;124(9):1465-1469.

26. Porres-Aguilar M, Rivera-Lebron B, Anaya-Ayala JE, Guerrero de León MC, Mukherjee D. Perioperative acute pulmonary embolism: a concise review with emphasis on multidisciplinary approach. Int J Angiol. 2020;29: (In Press; pending DOI). 